PROCEEDINGS OF THE

AMERICAN MATHEMATICAL SOCIETY

Volume 135, Number 10, October 2007, Pages 3349-3357

S 0002-9939(07)09012-0

Article electronically published on June 19, 2007

\title{
REAL HYPERSURFACES WITH CONSTANT PRINCIPAL CURVATURES IN THE COMPLEX HYPERBOLIC PLANE
}

\author{
JÜRGEN BERNDT AND JOSÉ CARLOS DÍAZ-RAMOS
}

(Communicated by Jon G. Wolfson)

\begin{abstract}
We classify real hypersurfaces with constant principal curvatures in the complex hyperbolic plane. It follows from this classification that all of them are open parts of homogeneous ones.
\end{abstract}

\section{INTRODUCTION}

Élie Cartan classified in [6] all connected hypersurfaces $M$ with constant principal curvatures in the real hyperbolic space $\mathbb{R} H^{n}, n \geq 3$. His classification exhibits two remarkable features. First, the number $g$ of distinct principal curvatures has an upper bound independent of the dimension $n$. In fact, Cartan showed that $g \leq 2$. Second, every connected real hypersurface with constant principal curvatures in $\mathbb{R} H^{n}$ is an open part of a homogeneous hypersurface. Therefore, assuming $M$ is complete, the constancy of principal curvatures is equivalent to the existence of a closed subgroup of the isometry group $G$ of $\mathbb{R} H^{n}$ such that $M$ is an orbit of $G$.

We are interested in the corresponding questions in the complex hyperbolic space $\mathbb{C} H^{n}, n \geq 2$. We briefly summarize the known facts so far. Cartan's argument for $g \leq 2$ in $\mathbb{R} H^{n}$ relies on the Gauß-Codazzi equations. The structure of these equations in $\mathbb{C} H^{n}$ is too complicated in general, but simplifies considerably for Hopf hypersurfaces. A real hypersurface $M$ in $\mathbb{C} H^{n}$ is a Hopf hypersurface if the Hopf foliation on $M$ is totally geodesic. The Hopf foliation on $M$ consists of the leaves of the one-dimensional distribution on $M$ that is obtained by rotating the normal bundle of $M$ into the tangent bundle of $M$ by means of the complex structure $J$ of $\mathbb{C} H^{n}$. If $\xi$ is a unit normal vector field on $M$, then $M$ is a Hopf hypersurface if and only if $J \xi_{p}$ is a principal curvature vector of $M$ at each point $p \in M$. The first author classified in [1] all connected Hopf hypersurfaces with constant principal curvatures in $\mathbb{C} H^{n}, n \geq 2$. It turns out that $g \in\{2,3\}$ and that $M$ is an open part of a homogeneous real hypersurface in $\mathbb{C} H^{n}$.

For some time it was believed that every homogeneous real hypersurface in $\mathbb{C} H^{n}$ is a Hopf hypersurface, a fact known to be true in the complex projective space $\mathbb{C} P^{n}$ according to Takagi's classification in [9]. Surprisingly, Lohnherr [8] constructed in

Received by the editors May 16, 2006.

2000 Mathematics Subject Classification. Primary 53C40; Secondary 53C55.

Key words and phrases. Homogeneous hypersurfaces, constant principal curvatures.

The second author has been supported by projects BFM 2003-02949 and PGIDIT 04 PXIC 20701 PN (Spain).

(C)2007 American Mathematical Society 3349

Reverts to public domain 28 years from publication 
his Ph.D. thesis an example of a homogeneous real hypersurface $W^{2 n-1}$ in $\mathbb{C} H^{n}$ which is not a Hopf hypersurface. Consider a horocycle $H$ in a totally geodesic $\mathbb{R} H^{2} \subset \mathbb{C} H^{2} \subset \mathbb{C} H^{n}$, and attach to each point $p \in H$ the totally geodesic $\mathbb{C} H^{n-1} \subset$ $\mathbb{C} H^{n}$ which is perpendicular to $H$ at $p$. The resulting ruled real hypersurface $W^{2 n-1}$ is a minimal homogeneous real hypersurface in $\mathbb{C} H^{n}$. An alternative Lie theoretic construction of $W^{2 n-1}$ has been presented by the first author in [2].

The first author and Brück constructed in 3 more examples of homogeneous real hypersurfaces in $\mathbb{C} H^{n}$ which are not Hopf hypersurfaces. Recently, the first author and Tamaru classified in [5] the cohomogeneity one actions on $\mathbb{C} H^{n}$ up to orbit equivalence. This of course provides the classification of homogeneous real hypersurfaces in $\mathbb{C} H^{n}$, and confirms that there are no further homogeneous real hypersurfaces in $\mathbb{C} H^{n}$ apart from the known ones. Any such hypersurface has constant principal curvatures with $g \in\{2,3,4,5\}$. The authors classified in [4] all connected real hypersurfaces in $\mathbb{C} H^{n}, n \geq 3$, with at most three distinct constant principal curvatures. A consequence of this classification is that any such hypersurface is an open part of a homogeneous real hypersurface in $\mathbb{C} H^{n}$. The methods developed in 4 do not work for the case $n=2$. The purpose of this paper is to settle this remaining case by a different method.

Theorem 1.1. Let $M$ be a connected real hypersurface in the complex hyperbolic plane $\mathbb{C} H^{2}$. Then $M$ has constant principal curvatures if and only if it is an open part of a homogeneous hypersurface in $\mathbb{C} H^{2}$, that is, $M$ is an open part of

(i) a geodesic hypersphere in $\mathbb{C H}^{2}$, or

(ii) a horosphere in $\mathbb{C} H^{2}$, or

(iii) a tube around a totally geodesic $\mathbb{C} H^{1} \subset \mathbb{C} H^{2}$, or

(iv) a tube around a totally geodesic $\mathbb{R} H^{2} \subset \mathbb{C} H^{2}$, or

(v) a ruled minimal real hypersurface $W^{3} \subset \mathbb{C} H^{2}$, or one of its equidistant hypersurfaces.

\section{Preliminaries}

Let $\mathbb{C} H^{2}$ be the complex hyperbolic plane equipped with the Fubini Study metric $\langle\cdot, \cdot\rangle$ of constant holomorphic sectional curvature -1 . We denote by $\bar{\nabla}$ and $\bar{R}$ the Levi Civita covariant derivative and the Riemannian curvature tensor of $\mathbb{C} H^{2}$, respectively, using the sign convention $\bar{R}_{X Y}=\left[\bar{\nabla}_{X}, \bar{\nabla}_{Y}\right]-\bar{\nabla}_{[X, Y]}$. Then

$$
\bar{R}_{X Y} Z=-\frac{1}{4}(\langle Y, Z\rangle X-\langle X, Z\rangle Y+\langle J Y, Z\rangle J X-\langle J X, Z\rangle J Y-2\langle J X, Y\rangle J Z),
$$

where $J$ is the complex structure of $\mathbb{C} H^{2}$. We also write $\bar{R}_{X Y Z W}=\left\langle\bar{R}_{X Y} Z, W\right\rangle$.

Let $M$ be a connected real hypersurface of $\mathbb{C} H^{2}$. We denote by $\nabla$ and $R$ the Levi Civita covariant derivative and the Riemannian curvature tensor of $M$, respectively. By $T M$ and $\nu M$ we denote the tangent bundle and the normal bundle of $M$, and by $\Gamma(T M)$ and $\Gamma(\nu M)$ we denote the module of all smooth vector fields tangent and normal to $M$, respectively. Let $X, Y, Z, W \in \Gamma(T M)$ and $\xi \in \Gamma(\nu M)$ be a (local) unit normal vector field on $M$.

The Levi Civita covariant derivatives of $M$ and $\mathbb{C} H^{2}$ are related by the Gauß formula

$$
\bar{\nabla}_{X} Y=\nabla_{X} Y+\langle S X, Y\rangle \xi
$$

where $S$ is the shape operator of $M$ with respect to $\xi$. The Weingarten formula is

$$
\bar{\nabla}_{X} \xi=-S X .
$$


The fundamental equations of second order of interest to us are the Gauß equation

$$
\bar{R}_{X Y Z W}=R_{X Y Z W}-\langle S Y, Z\rangle\langle S X, W\rangle+\langle S X, Z\rangle\langle S Y, W\rangle
$$

and the Codazzi equation

$$
\bar{R}_{X Y Z \xi}=\left\langle\left(\nabla_{X} S\right) Y-\left(\nabla_{Y} S\right) X, Z\right\rangle .
$$

We assume from now on that $M$ has constant principal curvatures. For each principal curvature $\lambda$ of $M$ we denote by $T_{\lambda}$ the distribution on $M$ formed by the principal curvature spaces of $\lambda$. By $\Gamma\left(T_{\lambda}\right)$ we denote the set of all smooth sections in $T_{\lambda}$, that is, all smooth vector fields on $M$ satisfying $S X=\lambda X$. The Codazzi equation readily implies

Lemma 2.1. For all $X \in \Gamma\left(T_{\lambda_{i}}\right), Y \in \Gamma\left(T_{\lambda_{j}}\right)$ and $Z \in \Gamma\left(T_{\lambda_{k}}\right)$ we have

$$
\bar{R}_{X Y Z \xi}=\left(\lambda_{j}-\lambda_{k}\right)\left\langle\nabla_{X} Y, Z\right\rangle-\left(\lambda_{i}-\lambda_{k}\right)\left\langle\nabla_{Y} X, Z\right\rangle \text {. }
$$

Putting $\lambda_{i}=\lambda_{k}$ in Lemma 2.1 and then interchanging $Y$ and $Z$ yields

Lemma 2.2. For all $X, Y \in \Gamma\left(T_{\lambda_{i}}\right)$ and $Z \in \Gamma\left(T_{\lambda_{j}}\right)$ with $\lambda_{i} \neq \lambda_{j}$ we have

$$
4\left(\lambda_{j}-\lambda_{i}\right)\left\langle\nabla_{X} Y, Z\right\rangle=\langle J Y, Z\rangle\langle X, J \xi\rangle+\langle J X, Y\rangle\langle Z, J \xi\rangle+2\langle J X, Z\rangle\langle Y, J \xi\rangle .
$$

\section{The Classification}

Let $M$ be a connected real hypersurface of $\mathbb{C} H^{2}$ with constant principal curvatures. Since our classification problem is of a local nature, we can assume that $M$ is orientable. Let $\xi$ be a global unit normal vector field on $M$ and denote by

$g$ the number of distinct constant principal curvatures of $M$. As an immediate consequence of Lemma 2.1 we have

Lemma 3.1. $g \geq 2$.

Proof. Suppose that $g=1$. Then Lemma 2.1 implies $\bar{R}_{X Y Z \xi}=0$ for all $X, Y, Z \in$ $\Gamma(T M)$. In particular, $0=4 \bar{R}_{J \xi Y Z \xi}=\langle J Y, Z\rangle$ for all $Y, Z \in \Gamma(T M)$, which means that $M$ is a totally real submanifold of $\mathbb{C} H^{2}$, and hence $\operatorname{dim} M \leq 2$, which is a contradiction to $\operatorname{dim} M=3$.

Proposition 3.2. If $g=2$, then $M$ is an open part of

(i) a geodesic hypersphere in $\mathbb{C} H^{2}$, or

(ii) a horosphere in $\mathbb{C} H^{2}$, or

(iii) a tube around a totally geodesic $\mathbb{C} H^{1} \subset \mathbb{C} H^{2}$, or

(iv) a tube of radius $r=\ln (2+\sqrt{3})$ around a totally geodesic $\mathbb{R} H^{2} \subset \mathbb{C} H^{2}$.

Proof. We denote by $\lambda_{i}$ the principal curvature of $M$ with multiplicity $i \in\{1,2\}$. Suppose there is a point $p \in M$ such that $J \xi_{p}$ is not a principal curvature vector. Then there exists an open neighborhood $N$ of $p$ in $M$ such that $J \xi=b_{1} U_{1}+b_{2} U_{2}$ for some unit vector fields $U_{i} \in \Gamma\left(T_{\lambda_{i}}\right)$ on $N$ and smooth functions $b_{i}: N \rightarrow \mathbb{R}$ with $b_{i}(q) \neq 0$ for all $q \in N$. As the rank of the distribution $T_{\lambda_{2}}$ is 2 , there exists a nonzero vector field $V \in \Gamma\left(T_{\lambda_{2}}\right)$ on $N$ such that $\left\langle V, U_{2}\right\rangle=0$. By construction we have $\langle J V, \xi\rangle=-\langle V, J \xi\rangle=0$ and $\langle J V, V\rangle=0$. From Lemma 2.1 we get $0=$ $4 \bar{R}_{U_{2} V U_{2} \xi}=3 b_{2}\left\langle J V, U_{2}\right\rangle$. Thus we have $\left\langle J V, U_{2}\right\rangle=0$ on $N$, and hence $b_{1}\left\langle J V, U_{1}\right\rangle=$ $-\left\langle V, b_{1} J U_{1}\right\rangle=\left\langle V, \xi+b_{2} J U_{2}\right\rangle=0$. This implies $\left\langle J V, U_{1}\right\rangle=0$ on $N$ as well. Altogether this shows that $V=0$ on $N$, which is a contradiction. We conclude that $J \xi_{p}$ is a principal curvature vector of $M$ at each point $p \in M$. The proposition then follows from [1]. 
In view of the above results we can assume $g=3$ from now on. The real hypersurfaces with constant principal curvatures in $\mathbb{C} H^{n}, n \geq 3$, have been classified by the authors in [4]. Unfortunately, the proof in [4] does not work for $n=2$. For this reason we need to develop here a different method for $n=2$.

We denote by $\lambda_{1}, \lambda_{2}$ and $\lambda_{3}$ the three distinct principal curvatures of $M$. As $\operatorname{dim} M=3$, each of these principal curvatures has multiplicity one. Let $U_{1}, U_{2}, U_{3}$ be a local orthonormal frame field on $M$ with $U_{i} \in \Gamma\left(T_{\lambda_{i}}\right)$, that is, $S U_{i}=\lambda_{i} U_{i}$. Since we are interested only in the local structure of $M$, we can assume without loss of generality that $U_{1}, U_{2}, U_{3}$ is a global orthonormal frame field on $M$. Then we can write

$$
J \xi=b_{1} U_{1}+b_{2} U_{2}+b_{3} U_{3}
$$

with the smooth functions $b_{i}: M \rightarrow \mathbb{R}$ defined by $b_{i}=\left\langle J \xi, U_{i}\right\rangle, i \in\{1,2,3\}$. Note that

$$
b_{1}^{2}+b_{2}^{2}+b_{3}^{2}=1 .
$$

In the following we assume that all indices are taken modulo 3 . As

$$
J U_{i}=\left\langle J U_{i}, U_{i+1}\right\rangle U_{i+1}-\left\langle J U_{i+2}, U_{i}\right\rangle U_{i+2}-b_{i} \xi,
$$

we get

$$
0=\left\langle U_{i}, \xi\right\rangle=\left\langle J U_{i}, J \xi\right\rangle=\left\langle J U_{i}, U_{i+1}\right\rangle b_{i+1}-\left\langle J U_{i+2}, U_{i}\right\rangle b_{i+2} .
$$

This gives a system of three linear equations, and we easily see that the vector $\left(b_{1}, b_{2}, b_{3}\right)$ is in the real span of $\left(\left\langle J U_{2}, U_{3}\right\rangle,\left\langle J U_{3}, U_{1}\right\rangle,\left\langle J U_{1}, U_{2}\right\rangle\right)$. From $b_{1}^{2}+b_{2}^{2}+b_{3}^{2}=$ 1 we get

$$
3=\sum_{i=1}^{3}\left\langle U_{i}, U_{i}\right\rangle=\sum_{i=1}^{3}\left\langle J U_{i}, J U_{i}\right\rangle=2\left(\left\langle J U_{2}, U_{3}\right\rangle^{2}+\left\langle J U_{3}, U_{1}\right\rangle^{2}+\left\langle J U_{1}, U_{2}\right\rangle^{2}\right)+1 .
$$

Thus $\left(\left\langle J U_{2}, U_{3}\right\rangle,\left\langle J U_{3}, U_{1}\right\rangle,\left\langle J U_{1}, U_{2}\right\rangle\right)$ is a unit vector in $\mathbb{R}^{3}$, and hence we must have $\left(b_{1}, b_{2}, b_{3}\right)= \pm\left(\left\langle J U_{2}, U_{3}\right\rangle,\left\langle J U_{3}, U_{1}\right\rangle,\left\langle J U_{1}, U_{2}\right\rangle\right)$. Without loss of generality we can assume that we have the plus sign (otherwise replace $\xi$ by $-\xi$ ). Thus we have proved

$$
\left\langle J U_{i}, U_{i+1}\right\rangle=b_{i+2} .
$$

We now introduce the following notation:

$$
d_{i}=\lambda_{i+1}-\lambda_{i+2}, x_{i}=\left\langle\nabla_{U_{i}} U_{i+1}, U_{i+2}\right\rangle .
$$

Putting $X=U_{i+1}, Y=U_{i+2}$ and $Z=U_{i}$ in Lemma 2.1, and using (3.1) and $b_{1}^{2}+b_{2}^{2}+b_{3}^{2}=1$, we obtain

$$
3 b_{i}^{2}=1+4 d_{i+2} x_{i+2}-4 d_{i+1} x_{i+1} .
$$

As $\nabla_{U_{i}} U_{i}=\left\langle\nabla_{U_{i}} U_{i}, U_{i+1}\right\rangle U_{i+1}+\left\langle\nabla_{U_{i}} U_{i}, U_{i+2}\right\rangle U_{i+2}$, Lemma 2.2 and (3.1) imply

$$
4 \nabla_{U_{i}} U_{i}=-3 b_{i} b_{i+2} d_{i+2}^{-1} U_{i+1}-3 b_{i} b_{i+1} d_{i+1}^{-1} U_{i+2} .
$$

We now calculate the differential $d b_{i}$ of the function $b_{i}=\left\langle U_{i}, J \xi\right\rangle$. We have $\bar{\nabla}_{U_{i}} J \xi=J \bar{\nabla}_{U_{i}} \xi=-\lambda_{i} J U_{i}$ and hence $\left\langle U_{i}, \bar{\nabla}_{U_{i}} J \xi\right\rangle=0$. This implies $d b_{i}\left(U_{i}\right)=$ $b_{i+1}\left\langle\nabla_{U_{i}} U_{i}, U_{i+1}\right\rangle+b_{i+2}\left\langle\nabla_{U_{i}} U_{i}, U_{i+2}\right\rangle$, and using (3.3) we get

$$
4 d b_{i}\left(U_{i}\right)=3 b_{1} b_{2} b_{3} d_{i} d_{i+1}^{-1} d_{i+2}^{-1} .
$$

Using (3.1) and (3.3) we get from

$$
\begin{aligned}
d b_{i}\left(U_{i+1}\right) & =b_{i+1}\left\langle\nabla_{U_{i+1}} U_{i}, U_{i+1}\right\rangle+b_{i+2}\left\langle\nabla_{U_{i+1}} U_{i}, U_{i+2}\right\rangle-\lambda_{i+1}\left\langle U_{i}, J U_{i+1}\right\rangle \\
& =-b_{i+1}\left\langle\nabla_{U_{i+1}} U_{i+1}, U_{i}\right\rangle-b_{i+2}\left\langle\nabla_{U_{i+1}} U_{i+2}, U_{i}\right\rangle+\lambda_{i+1}\left\langle J U_{i}, U_{i+1}\right\rangle
\end{aligned}
$$


that

$$
4 d b_{i}\left(U_{i+1}\right)=b_{i+2}\left(3 b_{i+1}^{2} d_{i+2}^{-1}+4 \lambda_{i+1}-4 x_{i+1}\right) .
$$

In a similar way we obtain

$$
4 d b_{i}\left(U_{i+2}\right)=b_{i+1}\left(3 b_{i+2}^{2} d_{i+1}^{-1}-4 \lambda_{i+2}+4 x_{i+2}\right) .
$$

From the Gauß equation and (3.1) we get

$$
4 R_{U_{i} U_{i+1} U_{i} U_{i+1}}=1-4 \lambda_{i} \lambda_{i+1}+3 b_{i+2}^{2} .
$$

On the other hand, by definition,

$$
R_{U_{i} U_{i+1} U_{i} U_{i+1}}=\left\langle\nabla_{U_{i}} \nabla_{U_{i+1}} U_{i}, U_{i+1}\right\rangle-\left\langle\nabla_{U_{i+1}} \nabla_{U_{i}} U_{i}, U_{i+1}\right\rangle-\left\langle\nabla_{\left[U_{i}, U_{i+1}\right]} U_{i}, U_{i+1}\right\rangle .
$$

The first term on the right-hand side can be calculated using (3.3), (3.5) and (3.6) as follows:

$$
\begin{aligned}
& 16\left\langle\nabla_{U_{i}} \nabla_{U_{i+1}} U_{i}, U_{i+1}\right\rangle \\
= & \left\langle\nabla_{U_{i}}\left(12 b_{i+1} b_{i+2} d_{i+2}^{-1} U_{i+1}-16 x_{i+1} U_{i+2}\right), U_{i+1}\right\rangle \\
= & 12\left(d b_{i+1}\left(U_{i}\right) b_{i+2}+b_{i+1} d b_{i+2}\left(U_{i}\right)\right) d_{i+2}^{-1}+16 x_{i} x_{i+1} \\
= & 9\left(b_{i+1}^{2} d_{i+1}^{-1}+b_{i+2}^{2} d_{i+2}^{-1}\right) b_{i}^{2} d_{i+2}^{-1}+12\left(x_{i}-\lambda_{i}\right)\left(b_{i+2}^{2}-b_{i+1}^{2}\right) d_{i+2}^{-1}+16 x_{i} x_{i+1} .
\end{aligned}
$$

The second and third term can be calculated in a similar way:

$$
\begin{aligned}
& 16\left\langle\nabla_{U_{i+1}} \nabla_{U_{i}} U_{i}, U_{i+1}\right\rangle \\
= & -12\left\langle\nabla_{U_{i+1}}\left(b_{i} b_{i+2} d_{i+2}^{-1} U_{i+1}-b_{i} b_{i+1} d_{i+1}^{-1} U_{i+2}, U_{i+1}\right\rangle\right. \\
= & -12\left(d b_{i}\left(U_{i+1}\right) b_{i+2}+b_{i} d b_{i+2}\left(U_{i+1}\right)\right) d_{i+2}^{-1}-9 b_{i}^{2} b_{i+1}^{2} d_{i}^{-1} d_{i+1}^{-1} \\
= & \left(12\left(x_{i+1}-\lambda_{i+1}\right)\left(b_{i+2}^{2}-b_{i}^{2}\right)-9\left(b_{i}^{2} d_{i}^{-1}+b_{i+2}^{2} d_{i+2}^{-1}\right) b_{i+1}^{2}\right) d_{i+2}^{-1}-9 b_{i}^{2} b_{i+1}^{2} d_{i}^{-1} d_{i+1}^{-1}, \\
& 16\left\langle\nabla_{\left[U_{i}, U_{i+1}\right]} U_{i}, U_{i+1}\right\rangle \\
= & -16\left\langle\nabla_{U_{i}} U_{i}, U_{i+1}\right\rangle^{2}-16\left\langle\nabla_{U_{i+1}} U_{i+1}, U_{i}\right\rangle^{2}+16 x_{i} x_{i+2}+16 x_{i+1} x_{i+2} \\
= & -9 b_{i}^{2} b_{i+2}^{2} d_{i+2}^{-2}-9 b_{i+1}^{2} b_{i+2}^{2} d_{i+2}^{-2}+16 x_{i} x_{i+2}+16 x_{i+1} x_{i+2} .
\end{aligned}
$$

From the previous equations we get by a straightforward calculation

$$
\begin{aligned}
0= & 8 \lambda_{i} \lambda_{i+1}-2-12 b_{i+2}^{2}+9 d_{i+2}^{-2} b_{i+2}^{2}\left(1-b_{i+2}^{2}\right) \\
& +8\left(x_{i} x_{i+1}-x_{i} x_{i+2}-x_{i+1} x_{i+2}\right) \\
& +6 d_{i+2}^{-1}\left(\left(b_{i+2}^{2}-b_{i+1}^{2}\right) x_{i}-\left(b_{i+2}^{2}-b_{i}^{2}\right) x_{i+1}+b_{i+1}^{2} \lambda_{i}-b_{i}^{2} \lambda_{i+1}\right) .
\end{aligned}
$$

The following proposition is the first crucial step towards the final classification.

Proposition 3.3. The functions $b_{1}, b_{2}, b_{3}$ are constant.

Proof. Inserting $b_{i}^{2}, b_{i+1}^{2}, b_{i+2}^{2}$ into (3.7) according to (3.2) gives

$$
\begin{aligned}
0= & 8 d_{i} d_{i+1} d_{i+2}^{-2}\left(x_{i}+x_{i+1}\right)^{2}+2 d_{i}\left(4+2 \lambda_{i} d_{i+2}^{-1}-d_{i+2}^{-2}\right) x_{i} \\
& -2 d_{i+1}\left(4-2 \lambda_{i+1} d_{i+2}^{-1}-d_{i+2}^{-2}\right) x_{i+1} \\
& -4\left(\lambda_{i}+\lambda_{i+1}\right) x_{i+2}+d_{i+2}^{-2}-2+4 \lambda_{i} \lambda_{i+1} .
\end{aligned}
$$


This leads to a system of three quadratic equations of the form

$$
\begin{aligned}
& \left(x_{1}+x_{2}\right)^{2}+\Lambda_{11} x_{1}+\Lambda_{12} x_{2}+\Lambda_{13} x_{3}=\Omega_{1}, \\
& \left(x_{1}+x_{3}\right)^{2}+\Lambda_{21} x_{1}+\Lambda_{22} x_{2}+\Lambda_{23} x_{3}=\Omega_{2}, \\
& \left(x_{2}+x_{3}\right)^{2}+\Lambda_{31} x_{1}+\Lambda_{32} x_{2}+\Lambda_{33} x_{3}=\Omega_{3}
\end{aligned}
$$

with some real constants $\Lambda_{i j}$ and $\Omega_{i}$. We introduce new variables $y_{1}, y_{2}, y_{3}$ by means of $x_{i}=-y_{i}+y_{i+1}+y_{i+2}$. Then the above system transforms into a system of three quadratic equations of the form

$$
4 y_{i}^{2}+\bar{\Lambda}_{i 1} y_{1}+\bar{\Lambda}_{i 2} y_{2}+\bar{\Lambda}_{i 3} y_{3}=\Omega_{i}, i \in\{1,2,3\} .
$$

This system has only finitely many solutions (see e.g. Corollary 7 on page 233 in [7). It follows that the system (3.8) has only finitely many solutions, and as the coefficients of the system are constant, each solution must be constant. Thus the functions $x_{1}, x_{2}, x_{3}$ are constant. From (3.2) we see that $b_{1}, b_{2}, b_{3}$ are constant.

Corollary 3.4. There exists a principal curvature $\lambda_{i}$ of $M$ such that the orthogonal projection of $J \xi_{p}$ onto $T_{\lambda_{i}}(p)$ is equal to zero for all $p \in M$.

Proof. As $b_{i}$ is constant, equation (3.4) shows that $b_{1} b_{2} b_{3}=0$, which implies the assertion.

In view of the previous corollary we may assume $b_{3}=0$. Moreover, if $b_{1}=0$ or $b_{2}=0$, then $M$ is a Hopf hypersurface. In this case it follows from the classification of Hopf hypersurfaces with constant principal curvatures in [1] that $M$ is an open part of a tube of radius $r \neq \ln (2+\sqrt{3})$ around a totally geodesic $\mathbb{R} H^{2} \subset \mathbb{C} H^{2}$. Therefore we assume $b_{1} \neq 0$ and $b_{2} \neq 0$ from now on.

Differentiating the constant function $b_{2}$ with respect to $U_{3}$ gives

$$
x_{3}=\lambda_{3}
$$

according to (3.5), and differentiating $b_{3}=0$ with respect to $U_{1}$ and $U_{2}$ gives

$$
4 x_{1}=4 \lambda_{1}+3 d_{2}^{-1} b_{1}^{2}, 4 x_{2}=4 \lambda_{2}-3 d_{1}^{-1} b_{2}^{2}
$$

according to (3.5) and (3.6). Inserting these expressions for $x_{1}, x_{2}, x_{3}$ into the two equations for $b_{1}^{2}$ and $b_{2}^{2}$ in (3.2), and subtracting the two resulting equations gives

$$
d_{1}^{-1} b_{2}^{2}-d_{2}^{-1} b_{1}^{2}=4 \lambda_{3}
$$

Together with the equation $b_{1}^{2}+b_{2}^{2}=1$ this leads to

$$
b_{1}^{2}=d_{2} d_{3}^{-1}\left(4 d_{1} \lambda_{3}-1\right), b_{2}^{2}=-d_{1} d_{3}^{-1}\left(4 d_{2} \lambda_{3}+1\right) .
$$

We now insert the expressions for $x_{1}$ and $x_{2}$ according to (3.10) and the ones for $b_{1}^{2}$ and $b_{2}^{2}$ according to (3.12) into the equation for $b_{3}^{2}=0$ in (3.2), which gives

$$
2 d_{1} \lambda_{1}-2 d_{2} \lambda_{2}+6\left(d_{2}-d_{1}\right) \lambda_{3}+1=0 .
$$

This equation is equivalent to

$$
\left(\lambda_{1}-\lambda_{2}\right)^{2}-\left(\lambda_{1}+\lambda_{2}-4 \lambda_{3}\right)^{2}=1-4 \lambda_{3}^{2} .
$$

We now multiply equation (3.7) for $i=2$ with $d_{1}$ and equation (3.7) for $i=3$ with $d_{2}$, and then subtract the two resulting equations, which gives

$$
\left(10 \lambda_{1}^{2}+10 \lambda_{2}^{2}+6 \lambda_{1} \lambda_{2}+1\right) \lambda_{3}=2\left(\lambda_{1}+\lambda_{2}\right)\left(4 \lambda_{3}^{2}+\lambda_{1} \lambda_{2}+1\right)+6 \lambda_{3}^{3} .
$$


We define $x=\lambda_{1}-\lambda_{2}$ and $y=\lambda_{1}+\lambda_{2}-4 \lambda_{3}$, which transforms the last two equations into

$x^{2}-y^{2}-1+4 \lambda_{3}^{2}=0, x^{2}\left(y+11 \lambda_{3}\right)-y^{3}+\lambda_{3} y^{2}+4\left(10 \lambda_{3}^{2}-1\right) y+2 \lambda_{3}\left(34 \lambda_{3}^{2}-7\right)=0$.

If $\lambda_{3}=0$ we immediately get $\lambda_{1}, \lambda_{2} \in\{ \pm 1 / 2\}$. Hence, we assume $\lambda_{3} \neq 0$. Inserting $x^{2}=y^{2}+1-4 \lambda_{3}^{2}$ into the second equation gives a quadratic equation in $y$, which easily leads to the possible solutions

$$
(x, y)=\left( \pm \sqrt{1-3 \lambda_{3}^{2}},-\lambda_{3}\right) \text { and }(x, y)=\left( \pm \frac{1}{4 \lambda_{3}}, \frac{1-8 \lambda_{3}^{2}}{4 \lambda_{3}}\right)
$$

where the first possibility only arises if $3 \lambda_{3}^{2} \leq 1$. Taking into account that $\lambda_{1}$ and $\lambda_{2}$ are different from $\lambda_{3}$, this eventually implies

$$
\lambda_{1}=\frac{1}{2}\left(3 \lambda_{3}-\sqrt{1-3 \lambda_{3}^{2}}\right), \lambda_{2}=\frac{1}{2}\left(3 \lambda_{3}+\sqrt{1-3 \lambda_{3}^{2}}\right),
$$

where we assume without loss of generality that $\lambda_{1}<\lambda_{2}$. Obviously, we get a solution only if $3 \lambda_{3}^{2} \leq 1$. If $\left|\lambda_{3}\right|=1 / 2$ or $\left|\lambda_{3}\right|=1 / \sqrt{3}$, then the three principal curvatures cannot be different. Suppose that $1 / 2<\left|\lambda_{3}\right|<1 / \sqrt{3}$. From (3.11) and (3.13) we get

$$
\frac{b_{1}^{2}}{2 \lambda_{3}\left(\lambda_{3}-\sqrt{1-3 \lambda_{3}^{2}}\right)}+\frac{b_{2}^{2}}{2 \lambda_{3}\left(\lambda_{3}+\sqrt{1-3 \lambda_{3}^{2}}\right)}=1 .
$$

If $1 / 2<\left|\lambda_{3}\right|<1 / \sqrt{3}$, elementary calculations show that $0<2 \lambda_{3}\left(\lambda_{3}-\sqrt{1-3 \lambda_{3}^{2}}\right)<$ 1 and $0<2 \lambda_{3}\left(\lambda_{3}+\sqrt{1-3 \lambda_{3}^{2}}\right)<1$. Therefore the last equation is the equation of an ellipse centered at the origin and with axes of length less than 1 . Obviously such an ellipse has no points of intersection with the circle $b_{1}^{2}+b_{2}^{2}=1$. This shows that $\left|\lambda_{3}\right|<1 / 2$.

Therefore, we have proved

Proposition 3.5. Let $M$ be a connected real hypersurface in $\mathbb{C} H^{2}$ with three distinct constant principal curvatures $\lambda_{1}, \lambda_{2}, \lambda_{3}$, and assume that $M$ is not a Hopf hypersurface. Then, with a suitable labelling of the principal curvatures, we have

$$
2\left|\lambda_{3}\right|<1,2 \lambda_{1}=3 \lambda_{3}-\sqrt{1-3 \lambda_{3}^{2}}, 2 \lambda_{2}=3 \lambda_{3}+\sqrt{1-3 \lambda_{3}^{2}} .
$$

This result is the second crucial step towards the final classification. We will now use Jacobi field theory to show that, under the assumptions of Proposition 3.5. one of the equidistant hypersurfaces to $M$ is an open part of the ruled minimal real hypersurface $W^{3}$.

For $r \in \mathbb{R}$ we define the smooth map $\Phi^{r}: M \rightarrow \mathbb{C} H^{2}, p \mapsto \Phi^{r}(p)=\exp _{p}\left(r \xi_{p}\right)$, where $\exp _{p}$ is the exponential map of $\mathbb{C} H^{2}$ at $p$. Geometrically this means that we assign to $p$ the point in $\mathbb{C} H^{2}$ which is obtained by travelling for the distance $r$ along the geodesic $c_{p}(t)=\exp _{p}\left(t \xi_{p}\right)$ in direction of the normal vector $\xi_{p}$ (for $r>0$; for $r<0$ one sets off in direction $-\xi_{p}$; and for $r=0$ there is no movement at all). For $v \in T_{p} M$ we denote by $B_{v}$ the parallel vector field along the geodesic $c_{p}$ with $B_{v}(0)=v$, and by $\zeta_{v}$ the Jacobi field along $c_{p}$ with $\zeta_{v}(0)=v$ and $\zeta_{v}^{\prime}(0)=-S_{p} v$. Note that $\zeta_{v}$ is the unique solution of the linear differential equation

$$
4 \zeta_{v}^{\prime \prime}-\zeta_{v}-3\left\langle\zeta_{v}, J \dot{c}_{p}\right\rangle J \dot{c}_{p}=0, \zeta_{v}(0)=v, \zeta_{v}^{\prime}(0)=-S_{p} v,
$$


where $\dot{c}_{p}$ denotes the tangent vector field of $c_{p}$ and the prime ' indicates the covariant derivative of a vector field along $c_{p}$. For $v \in T_{\lambda_{i}}(p)$ we have the explicit expression

$$
\zeta_{v}(t)=f_{i}(t) B_{v}(t)+\langle v, J \xi\rangle g_{i}(t) J \dot{c}_{p}(t)
$$

with

$$
\begin{aligned}
& f_{i}(t)=\cosh (t / 2)-2 \lambda_{i} \sinh (t / 2), \\
& g_{i}(t)=(\cosh (t / 2)-1)\left(1+2 \cosh (t / 2)-2 \lambda_{i} \sinh (t / 2)\right) .
\end{aligned}
$$

Finally, we define a vector field $\eta^{r}$ along the map $\Phi^{r}$ by $\eta_{p}^{r}=\dot{c}_{p}(r)$. The relation between the map $\Phi^{r}$, the vector field $\eta^{r}$ and the Jacobi field $\zeta_{v}$ is given by

$$
\zeta_{v}(r)=\Phi_{*}^{r} v, \zeta_{v}^{\prime}(r)=\bar{\nabla}_{v} \eta^{r},
$$

where $\Phi_{*}^{r}$ denotes the differential of $\Phi^{r}$. We will see that there exists a real number $r \in \mathbb{R}$ such that the map $\Phi^{r}$ has constant rank 3 and the image is locally a minimal real hypersurface $\mathcal{W}$. We then use the equation $\zeta_{v}^{\prime}(r)=\bar{\nabla}_{v} \eta^{r}$ to obtain some information about the second fundamental form of $\mathcal{W}$.

The following result was proved in [4].

Theorem 3.6. Let $M$ be a 3 -dimensional connected submanifold in $\mathbb{C} H^{2}$. Assume that there exists a unit vector field $Z$ tangent to the maximal holomorphic subbundle of $T M \subset T \mathbb{C} H^{2}$ such that the second fundamental form II of $M$ is given by the trivial bilinear extension of $2 I I(Z, J \xi)=\xi$ for all $\xi \in \Gamma(\nu M)$. Then $M$ is holomorphically congruent to an open part of the ruled minimal submanifold $W^{3}$.

If $\lambda_{3}=0$, then $\lambda_{1}=-1 / 2$ and $\lambda_{2}=1 / 2$, and (3.12) implies $2 b_{1}^{2}=2 b_{2}^{2}=1$. Thus, if we define $Z=-b_{1} U_{1}+b_{2} U_{2}$, we see from Theorem 3.6 that $M$ is holomorphically congruent to an open part of the ruled minimal hypersurface $W^{3}$.

If $\lambda_{3} \neq 0$, then $0<2\left|\lambda_{3}\right|<1$ by Proposition 3.5. Thus we can write $2 \lambda_{3}=$ $\tanh (r / 2)$ with some nonzero real number $r$. Using the equation $\Phi_{*}^{r} v=\zeta_{v}(r)$ and the explicit expression for the Jacobi fields, we obtain

$$
\Phi_{*}^{r} U_{3}(p)=\operatorname{sech}(r / 2) B_{U_{3}(p)}(r)
$$

and

$$
\left(\begin{array}{l}
\Phi_{*}^{r} U_{1}(p) \\
\Phi_{*}^{r} U_{2}(p)
\end{array}\right)=D(r)\left(\begin{array}{l}
B_{U_{1}(p)}(r) \\
B_{U_{2}(p)}(r)
\end{array}\right)
$$

with

$$
D(t)=\left(\begin{array}{cc}
f_{1}(t)+b_{1}^{2} g_{1}(t) & b_{1} b_{2} g_{1}(t) \\
b_{1} b_{2} g_{2}(t) & f_{2}(t)+b_{2}^{2} g_{2}(t)
\end{array}\right)
$$

for all $p \in M$. As $\operatorname{det}(D(r))=\operatorname{sech}^{3}(r / 2)$, we can now conclude that $\Phi_{*}^{r}$ has maximal rank everywhere. This means that for every point in $M$ there exists an open neighborhood $\mathcal{V}$ around that point such that $\mathcal{W}=\Phi^{r}(\mathcal{V})$ is an embedded real hypersurface of $\mathbb{C} H^{2}$ and $\Phi^{r}: \mathcal{V} \rightarrow \mathcal{W}$ is a diffeomorphism. Let $\mathcal{V}$ be such an open neighborhood. We fix a point $p \in \mathcal{V}$ and define $q=\Phi^{r}(p) \in \mathcal{W}$. The tangent space $T_{q} \mathcal{W}$ of $\mathcal{W}$ at $q$ is obtained by parallel translation of $T_{p} \mathcal{V}$ along the geodesic $c_{p}$ from $p=c_{p}(0)$ to $q=c_{p}(r)$, and $\eta_{p}^{r}$ is a unit normal vector of $\mathcal{W}$ at $q$.

For the shape operator $S^{r}$ of $\mathcal{W}$ we have $S_{\eta_{p}^{r}}^{r} \Phi_{*}^{r} v=-\bar{\nabla}_{v} \eta^{r}=-\zeta_{v}^{\prime}(r)$ for all $v \in T_{p} M$. Since $f_{3}^{\prime}(r)=0$, we immediately get

$$
S_{\eta_{p}^{r}}^{r} B_{U_{3}(p)}(r)=0,
$$


and by putting $v=U_{1}(p)$ and $v=U_{2}(p)$ we get

$$
\left(\begin{array}{c}
S_{\eta_{p}^{r}}^{r} B_{U_{1}(p)}(r) \\
S_{\eta_{p}^{r}}^{r} B_{U_{2}(p)}(r)
\end{array}\right)=C(r)\left(\begin{array}{c}
B_{U_{1}(p)}(r) \\
B_{U_{2}(p)}(r)
\end{array}\right)
$$

with $C(r)=-D^{\prime}(r) D(r)^{-1}$. A tedious calculation shows that $(\operatorname{det}(D))^{\prime}(r)=0$ and $\operatorname{det}\left(D^{\prime}(r)\right)=-\operatorname{sech}^{3}(r / 2) / 4$, which implies

$$
\operatorname{det}(C(r))=\frac{\operatorname{det}\left(D^{\prime}(r)\right)}{\operatorname{det}(D(r))}=-\frac{1}{4} \quad \text { and } \quad \operatorname{tr}(C(r))=-\frac{(\operatorname{det}(D))^{\prime}(r)}{\operatorname{det}(D(r))}=0 .
$$

From this we easily see that the eigenvalues of $C(r)$ are $\pm 1 / 2$. Altogether we now get that $\mathcal{W}$ has three distinct constant principal curvatures $0,+1 / 2$ and $-1 / 2$. According to the classification of real Hopf hypersurfaces in [1] we see that $\mathcal{W}$ cannot be a Hopf hypersurface. As $U_{3}(p)$ belongs to the maximal holomorphic subspace of $T_{p} M$ and parallel translation in $\mathbb{C} H^{2}$ along the geodesic $c_{p}$ commutes with the complex structure $J$, the vector $B_{U_{3}(p)}(r)$ belongs to the maximal holomorphic subspace of $T_{q} \mathcal{W}$. As we have seen above, $B_{U_{3}(p)}(r)$ is a principal curvature vector corresponding to the principal curvature 0 . It follows that we can write $J \eta_{p}^{r}$ as a linear combination of principal curvature vectors corresponding to the principal curvatures $\pm 1 / 2$. Applying (3.12) to $\mathcal{W}$ instead of $M$ we see that we can write $J \eta^{r}=\left(X_{+}+X_{-}\right) / \sqrt{2}$ with suitable unit vector fields $X_{+}$and $X_{-}$corresponding to the principal curvatures $+1 / 2$ and $-1 / 2$, respectively. Defining $Z=\left(X_{+}-X_{-}\right) / \sqrt{2}$ we get that the second fundamental form of $\mathcal{W}$ satisfies the formula of Theorem 3.6. It follows that $\mathcal{W}$ is holomorphically congruent to an open part of the ruled real hypersurface $W^{3}$. From this we eventually conclude that $M$ is holomorphically congruent to an open part of an equidistant hypersurface to $W^{3}$.

This finishes the proof of Theorem 1.1

\section{REFERENCES}

1. J. Berndt, Real hypersurfaces with constant principal curvatures in complex hyperbolic space, J. Reine Angew. Math. 395 (1989), 132-141. MR983062 (90d:53062)

2. J. Berndt, Homogeneous hypersurfaces in hyperbolic spaces, Math. Z. 229 (1998), 589-600. MR 1664778 (2001c:53065)

3. J. Berndt, M. Brück, Cohomogeneity one actions on hyperbolic spaces, J. Reine Angew. Math. 541 (2001), 209-235. MR.1876290(2002j:53059)

4. J. Berndt, J.C. Díaz-Ramos, Real hypersurfaces with constant principal curvatures in complex hyperbolic space, J. London Math. Soc., (2) 74 (2006), 778-798. MR983062 (90d:53062)

5. J. Berndt, H. Tamaru, Cohomogeneity one actions on noncompact symmetric spaces of rank one, Trans. Amer. Math. Soc., 359 (2007), 3425-3438.

6. É. Cartan, Familles de surfaces isoparamétriques dans les espaces à courbure constante, Ann. Mat. Pura Appl., IV. Ser. 17 (1938), 177-191. MR.1553310

7. D. Cox, J. Little, D. O'Shea, Ideals, varieties, and algorithms: an introduction to computational algebraic geometry and commutative algebra, Springer, New York, 1992. MR1189133 (93j:13031)

8. M. Lohnherr, On ruled real hypersurfaces of complex space forms, Ph.D. Thesis, University of Cologne, 1998.

9. R. Takagi, On homogeneous real hypersurfaces in a complex projective space, Osaka J. Math. 10 (1973), 495-506. MR0336660 (49:1433)

Department of Mathematics, University College, Cork, Ireland

E-mail address: j.berndt@ucc.ie

Department of Mathematics, University College, Cork, Ireland

E-mail address: jc.diazramos@ucc.ie 